\title{
The safety of isoniazid tuberculosis preventive treatment in pregnant and postpartum women: systematic review and meta-analysis
}

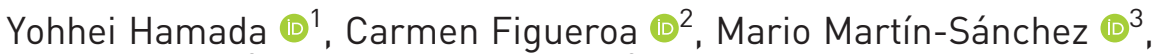 \\ Dennis Falzon ${ }^{2}$ and Avinash Kanchar ${ }^{2}$ \\ Affiliations: ${ }^{1}$ Research Institute of Tuberculosis, Japan Anti-tuberculosis Association, Tokyo, Japan. ${ }^{2}$ Global \\ TB Program (GTB), World Health Organization, Geneva, Switzerland. ${ }^{3}$ Preventive Medicine and Public \\ Health Training Unit, Parc de Salut Mar-Pompeu Fabra University-Agència de Salut Pública de Barcelona \\ (PSMar-UPF-ASPB), Barcelona, Spain.
}

Correspondence: Yohhei Hamada, Research Institute of Tuberculosis, Japan Anti-tuberculosis Association, Matsuyama 3-1-24, Kiyose, Tokyo 204-8533, Japan. E-mail: yohei.hamada0Agmail.com

@ERSpublications

Studies report conflicting links between isoniazid preventive therapy (IPT) and adverse pregnancy outcomes. Given known harms of active TB in pregnancy, the findings do not support systematic deferral of IPT until postpartum. We need more safety research. http://bit.ly/2R0Wc3G

Cite this article as: Hamada Y, Figueroa C, Martín-Sánchez M, et al. The safety of isoniazid tuberculosis preventive treatment in pregnant and postpartum women: systematic review and meta-analysis. Eur Respir J 2020; 55: 1901967 [https://doi.org/10.1183/13993003.01967-2019].

\section{ABSTRACT}

Background: The World Health Organization (WHO) recommends tuberculosis (TB) preventive treatment for high-risk groups. Isoniazid preventive therapy (IPT) has been used globally for this purpose for many years, including in pregnancy. This review assessed current knowledge about the safety of IPT in pregnancy.

Methods: We searched PubMed, Embase, CENTRAL, Global Health Library and HIV and TB-related conference abstracts, until May 15, 2019, for randomised controlled trials (RCTs) and non-randomised studies (NRS) where IPT was administered to pregnant women. Outcomes of interest were: 1) maternal outcomes, including permanent drug discontinuation due to adverse drug reactions, any grade 3 or 4 drugrelated toxic effects, death from any cause and hepatotoxicity; and 2) pregnancy outcomes, including in utero fetal death, neonatal death or stillbirth, preterm delivery/prematurity, intrauterine growth restriction, low birth weight and congenital anomalies. Meta-analyses were conducted using a random-effects model.

Results: After screening 1342 citations, nine studies (of 34 to 51942 participants) met inclusion criteria. We found an increased likelihood of hepatotoxicity among pregnant women given IPT (risk ratio 1.64, 95\% CI 0.78-3.44) compared with no IPT exposure in one RCT. Four studies reported on pregnancy outcomes comparing IPT exposure to no exposure among pregnant women with HIV. In one RCT, adverse pregnancy outcomes were associated with IPT exposure during pregnancy (odds ratio (OR) 1.51, 95\% CI 1.09-2.10), but three NRS showed a protective effect.

Conclusions: We found inconsistent associations between IPT and adverse pregnancy outcomes. Considering the grave consequences of active TB in pregnancy, current evidence does not support systematic deferral of IPT until postpartum. Research on safety is needed.

This article has supplementary material available from erj.ersjournals.com

Received: 9 Oct 2019 | Accepted after revision: 23 Nov 2019

The content of this work is copyright of the authors or their employers. Design and branding are copyright OERS 2020 


\section{Introduction}

Pregnant women with HIV have a high risk of acquiring tuberculosis (TB), which can have severe consequences for both mother and fetus [1]. Isoniazid has a well-documented safety profile established from its long history of use in pregnant and breastfeeding mothers treated for both latent and active TB.

The 2011 World Health Organization (WHO) guidelines recommend isoniazid preventive therapy (IPT) in people living with HIV regardless of pregnancy [2]. These guidelines and the 2018 guideline on latent tuberculosis infection (LTBI) advise caution and clinical judgement when deciding the best time to start LTBI treatment in pregnant women [3]. Pregnancy and the postpartum period is a risk factor for drug-induced hepatotoxicity [4] and, although evidence is insufficient, the WHO encourages clinical monitoring as well as baseline liver function tests where feasible for these groups [3].

A recent clinical trial reported more frequent adverse pregnancy outcomes among women with HIV exposed to IPT during gestation [5]. The study also reported higher frequency of maternal adverse events (AEs) than expected. To date there has been no systematic review which has investigated the safety of IPT among pregnant women. Therefore, we have conducted this systematic review to assess the safety of IPT in pregnant and postpartum women compared to other preventive treatment regimens or no treatment.

\section{Material and methods}

\section{Search strategy}

We performed a systematic review and meta-analysis using the Preferred Reporting Items for Systematic Reviews and Meta-analyses (PRISMA) [6] and Meta-analysis Of Observational Studies in Epidemiology (MOOSE) [7]. The protocol for this review is registered on PROSPERO (CRD42019136065, www.crd.york. ac.uk/prospero/).

We searched the following databases from inception to May 15, 2019: MEDLINE (PubMed), Embase, the Cochrane Central Register of Controlled Trials (CENTRAL) and the Global Health Library. Furthermore, we reviewed databases listing ongoing randomised controlled trials (RCTs) through ClinicalTrials.gov and the WHO International Clinical Trials Registry Platform. We developed the search strategy in consultation with a librarian (see appendix 1 in the supplementary material) and searched the following major HIV and TB conferences: the International AIDS Conference, the International AIDS Society (IAS) Conference on HIV Science, the Conference on Retroviruses and Opportunistic Infections, the UNION World Conference on Lung Health, the European Respiratory Society (ERS) Congress and the American Thoracic Society (ATS) Conference. The International AIDS Conference and the IAS Conference of HIV Science were searched for all available years (2002-2019), while, for the Conference on Retroviruses and Opportunistic Infections, the UNION World Conference on Lung Health, the ERS Congress and the ATS Conference, only conferences from the last 3 years were searched. We did not impose any language or geographic restrictions. Bibliographies of included articles were screened and we contacted experts and authors of relevant studies to retrieve relevant study information.

\section{Study eligibility and data extraction}

Two reviewers independently screened study titles and abstracts identified by the search for inclusion. The full text was then screened by the same pair of reviewers and eligibility was assessed. Any disagreements were resolved through discussion.

Studies were included when 1) the study population included pregnant or postpartum women (defined as within 12 months of delivery) regardless of HIV status; 2) the intervention was preventive treatment with daily isoniazid alone for 6 months or longer; 3) the comparator was another preventive treatment regimen or no preventive treatment (including deferred provision until postpartum in a comparison group); 4) the outcomes included permanent drug discontinuation due to an adverse drug reaction, any Grade 3 or 4 drug-related toxic effect, death from any cause, hepatotoxicity, in utero fetal death, neonatal death, preterm delivery/prematurity, intrauterine growth restriction, low birth weight or congenital anomalies; and 5) the study design was an RCT or a non-randomised study (NRS). We initially intended to exclude studies without a comparison group; however, we eventually included them due to the limited number of studies identified. We excluded studies that included participants with active TB or those who were exposed to multidrug-resistant TB (MDR-TB) or isoniazid-resistant TB.

Data were extracted independently by two reviewers using standardised extraction forms, as follows: study design, total duration and date of study, and study context (setting, location); number of participants, age, race, ethnicity, body mass index (BMI), body weight, education history, HIV status (plus antiretroviral status, CD4 count and viral load), obstetric history, inclusion criteria, exclusion criteria, comorbidities, results of tuberculin skin tests and interferon $-\gamma$ releasing assays, and contact history; type of intervention, 
comparison, concomitant medications and outcomes. Any disagreements were resolved by consensus and authors were contacted for missing data.

\section{Quality of individual studies and evidence assessment}

For the risk of bias in individual studies, we used the revised Cochrane Risk-of-Bias tool (RoB2) for RCTs [8] and the Risk Of Bias In Non-randomized Studies of Interventions (ROBINS-I) tool for NRS [9]. Grading of recommendations assessment, development, and evaluation (GRADE) methodology was used to assess and appraise the quality of evidence for each outcome across all studies [10]. We made an overall judgement on the quality of evidence across RCTs and NRS separately. When ROBINS-I was used, the initial rating of the certainty of evidence was assigned as high rather than low and was subsequently rated down, as recently recommended by the GRADE working group [11].

\section{Statistical analysis and meta-analysis}

Relative risks for dichotomous data were presented with $95 \%$ confidence intervals (CI). Meta-analysis was conducted with a random effects model using the method of DERSimonian and LAIRD [12] if the included studies were sufficiently clinically homogenous. When at least one study included zero events in one group, the Mantel-Haenszel method was used without continuity correction. Due to inconsistency in the direction of effect indicating a significant heterogeneity by study type, data from RCTs and NRS was not pooled. For NRS, adjusted estimates were pooled and, if unavailable, unadjusted estimates were pooled; however, unadjusted and adjusted estimates were not pooled together. Data was presented by HIV status, by pregnancy or postpartum status and by preventive treatment regimen given to the control group; however, the limited number of studies precluded meta-analysis by subgroup. Forest plots were used to visually assess heterogeneity among the included trials. Unfortunately, the small number of studies precluded a sensitivity analysis.

\section{Results}

From 1342 records identified, nine studies met our inclusion criteria, including two conference abstracts (figure 1) [4, 5, 13-19]. Six studies included only women with HIV $[5,13,15-17,19]$ and three included very few or no HIV-positive women $[4,14,18]$. Of the six studies among HIV-positive women, five were conducted in African countries [13, 15-17, 19] and one was conducted in multiple countries with high TB prevalence $(\geqslant 60$ per 100000 population) [5]. Eight studies were NRS, with three reporting data among women who were enrolled in trials of different preventive treatment regimens and who became pregnant during those trials $[14,17,19]$. Four included pregnant women started on IPT $[4,13,15,16]$ and one included postpartum women [18]. The remaining study was a RCT comparing pregnant women with HIV who started 6 months of IPT immediately upon enrolment and those who deferred it until 12 weeks postpartum [5]. Study characteristics are summarised in table 1.

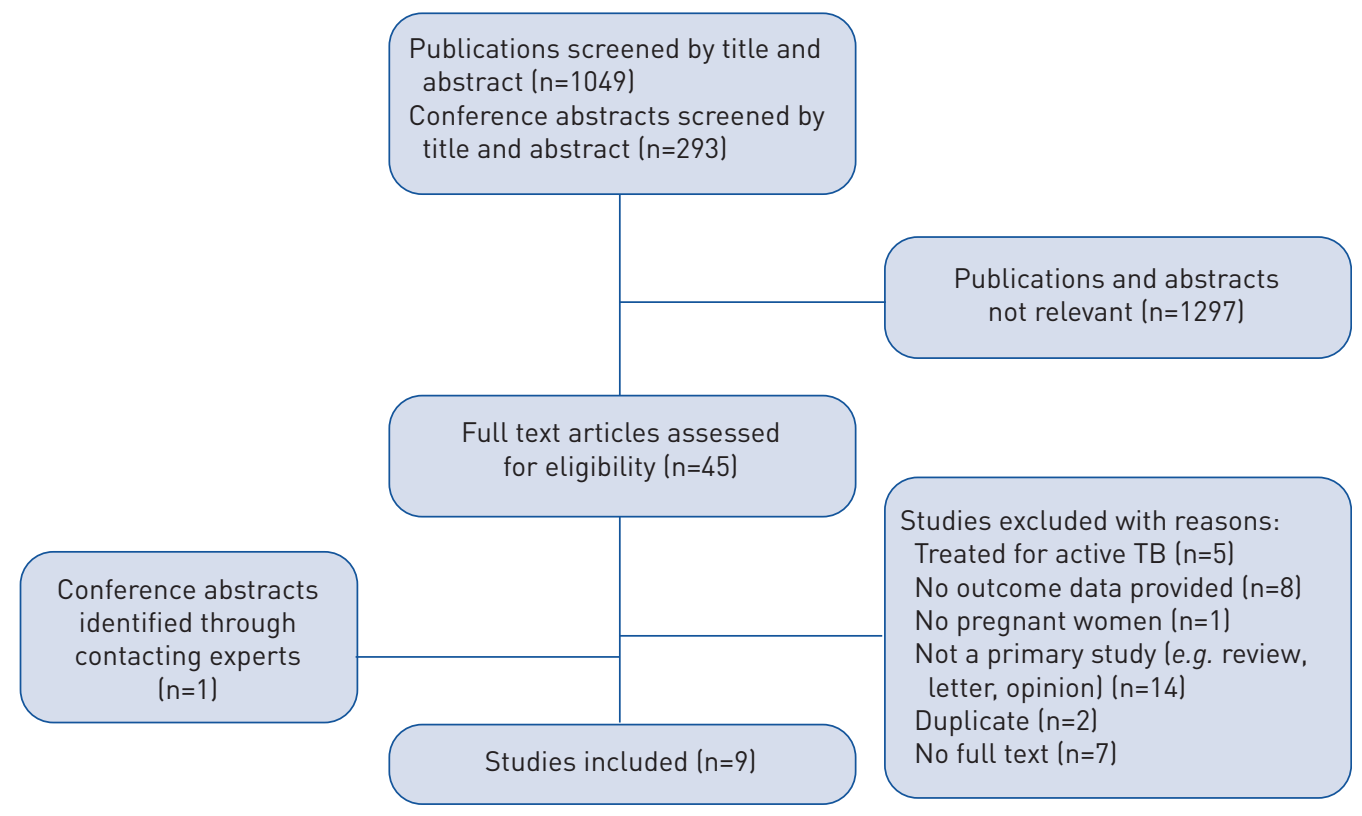

FIGURE 1 PRISMA flow diagram for the review. 
TABLE 1 Characteristics of included studies

\begin{tabular}{|c|c|c|c|c|c|}
\hline First author & Study design & Setting & Population & Isoniazid arm & Comparison \\
\hline GuPTA et al. [5] & RCT & $\begin{array}{l}\text { Eight countries with high } \\
\text { TB prevalence ( } \geqslant 60 \text { per } \\
100000 \text { population) }\end{array}$ & $\begin{array}{l}\text { HIV-infected pregnant } \\
\text { women }(\geqslant 18 \text { years old, } \\
14-34 \text { weeks gestation and } \\
99.8-100 \% \text { on cART) }\end{array}$ & $\begin{array}{l}\text { Immediate isoniazid, } \\
\text { started at study entry } \\
\text { and continued for } \\
28 \text { weeks ( } n=477 \text { ) }\end{array}$ & $\begin{array}{l}\text { Deferred isoniazid, } \\
\text { started at } 12 \text { weeks } \\
\text { postpartum and } \\
\text { continued for } \\
28 \text { weeks ( } \mathrm{n}=479 \text { ) }\end{array}$ \\
\hline Franks et al. [4] & $\begin{array}{c}\text { Retrospective } \\
\text { cohort }\end{array}$ & A clinic, USA & $\begin{array}{l}\text { Women enrolled during the } \\
\text { first } 18 \text { months of the } \\
\text { prenatal IPT programme }\end{array}$ & $\begin{array}{l}\text { 6-12 months of } \\
\text { isoniazid ( } n=3681)\end{array}$ & $\begin{array}{l}\text { No control group } \\
\text { who were given no or } \\
\text { other regimens }\end{array}$ \\
\hline $\begin{array}{l}\text { KALK et al. } \\
{[15]^{\#}}\end{array}$ & $\begin{array}{l}\text { Retrospective } \\
\text { cohort }\end{array}$ & $\begin{array}{l}\text { Routine electronic clinical } \\
\text { information systems from } \\
\text { public sector health } \\
\text { facilities, Western Cape, } \\
\text { South Africa }\end{array}$ & $\begin{array}{l}\text { HIV-infected women on or } \\
\text { initiating cART during } \\
\text { pregnancy ( } 41.8 \% \text { newly } \\
\text { initiated on cART and the } \\
\text { rest already on cART) }\end{array}$ & $\begin{array}{l}\text { Isoniazid duration } \\
\text { unknown, based on } \\
\text { "prescription" in the } \\
\text { electronic record } \\
\text { (n=10715) }\end{array}$ & $\begin{array}{l}\text { No treatment } \\
(n=41227)\end{array}$ \\
\hline Moro et al. [14] & $\begin{array}{l}\text { Sub-analysis of } \\
\text { two RCTs }\end{array}$ & $\begin{array}{l}\text { TBTC } 26 \text { and } 33 \text { IUSA, } \\
\text { Canada, Brazil, Spain, }\end{array}$ & $\begin{array}{l}\text { Women who became } \\
\text { pregnant during the trial }\end{array}$ & $\begin{array}{c}9 \text { months isoniazid } \\
(n=56)\end{array}$ & $\begin{array}{l}\text { 3HP }(n=31), \text { no } \\
\text { exposure }(n=39)\end{array}$ \\
\hline
\end{tabular}

$\begin{array}{cc}\text { Salazar-Austin } & \text { Prospective } \\ \text { et al. } \text { [13] } & \text { cohort }\end{array}$

TAYLOR et al. [17]

TIAM et al. [16]

\author{
Sub-analysis of \\ RCT
}

Peru, South Africa and Hong Kongl

Antenatal clinics and obstetrics wards at a Hospital, South Africa

Clinics, Botswana

wo hospital-based maternal and child health clinics, Lesotho

$$
\text { Prospective }
$$

pregnant during the trial

Pregnant women with HIV $l \geqslant 18$ years old, $66-78 \%$ on (ART)

Women with HIV $1 \geqslant 18$ years old, became pregnant during the trial, $37 \%$ on CART and the rest on AZT or AZT/3TC)

Pregnant women $(\geqslant 14$ years old with HIV, presented for their first antenatal clinic visit irrespective of their gestational age, $36.2 \%$ on CART and the rest on AZT prophylaxis)
6 months isoniazid

Imedian gestational age at initiation

25 weeks, IQR 20

30 weeks) ( $n=71)$

6-36 months isoniazid ( $n=103$ )

6 months of isoniazid (n=124)
No isoniazid $(n=84)$

No exposure $(\mathrm{n}=93)$

No control group who were given no or other regimens

RCT: randomised controlled trial; TB: tuberculosis; TBTC: Tuberculosis Trials Consortium; LTBI: latent tuberculosis infection; IPT: isoniazid preventive therapy; cART: combination antiretroviral therapy; AZT: zidovudine; 3TC: lamivudine; IQR: interquartile range; 3HP: 3-month weekly rifapentine plus isoniazid. ${ }^{\#}$ : conference abstracts.

\section{Maternal outcomes}

Four studies reported data on hepatotoxicity in pregnant women with HIV [5, 15-17], five studies reported deaths [5, 13, 15-17], two studies reported Grade 3 or 4 AEs [5, 16] and one study reported treatment discontinuations [5] (supplementary tables S3-S5). The RCT by GuPTA et al. [5] reported the highest frequency of hepatotoxicity (6.1\% in the immediate IPT arm and $7.1 \%$ in the deferred IPT arm), while KARL et al. [15] reported only $0.3 \%$ and two other NRS reported none. Frequency of deaths ranged from none to $2 \%$ across studies.

GuPTA et al. [5] provided data on hepatotoxicity compared to placebo by restricting to "events that occurred until 3 months postpartum", before the control group was started on IPT (table 2). On analysis, the frequency of hepatotoxicity was higher in women living with HIV who were given IPT during 
TABLE 2 Hepatotoxicity in pregnant women living with HIV

\begin{tabular}{|c|c|c|c|}
\hline Study & IPT & Control & Risk ratio $(95 \% \mathrm{CI})$ \\
\hline GuPTA et al. [5] & 18 out of $477(3.8 \%)^{\#}$ & $\begin{array}{c}\text { Placebo } \\
11 \text { out of } 479(2.3 \%)^{\#}\end{array}$ & $1.64(0.78-3.44)$ \\
\hline KALK et al. [15] & 30 out of $10715(0.3 \%)$ & $\begin{array}{c}\text { No treatment } \\
114 \text { out of } 41227(0.3 \%)\end{array}$ & $1.01(0.68-1.51)$ \\
\hline TIAM et al. [16] & 0 out of $124(0 \%)$ & NA & NA \\
\hline TAYLOR et al. [17] & 0 out of $103(0 \%)$ & NA & NA \\
\hline
\end{tabular}

IPT: isoniazid preventive therapy; $\mathrm{Cl}$ : confidence interval; NA: not available. ${ }^{\text {\# }}$ : the analysis was restricted to events that occurred until 3 months postpartum. Some women were still on IPT and were censored.

pregnancy (18 out of $477,3.8 \%$ ) compared to those given a placebo (11 out of $479,2.3 \%$ ); however, this difference was not statistically significant (risk ratio 1.64, 95\% CI 0.78-3.44) [5]. KALK et al. [15] did not find any difference in frequency of hepatotoxicity between the two groups.

Three studies reported maternal death in pregnant women with HIV who received IPT compared to those who did not $[5,13,17]$ (table 3 ). The RCT did not show a statistically significant difference in the risk of death between the two groups [5]. Meta-analysis of two NRS suggested a lower risk of death in pregnant women with HIV given IPT (risk ratio $0.65,95 \%$ CI 0.39-1.07).

The RCT [5] provided data on Grade 3 or 4 AEs and treatment discontinuations in pregnant women with HIV given IPT compared to those given a placebo. There was no statistical difference in the frequency of treatment discontinuation between the two groups (2.3\% versus $1.7 \%$; risk ratio $1.38,95 \%$ CI $0.56-3.40$ ). However, there was a higher risk of Grade 3 or 4 AEs in participants given IPT (7.1\% versus 4.6\%; risk ratio $1.55,95 \%$ CI $0.92-2.61$ ).

For HIV-negative pregnant women, Moro et al. [14] reported data on Grade 3 or 4 AEs, hepatotoxicity and deaths in pregnant women on IPT $(n=56)$ and those on 3-month weekly rifapentine plus isoniazid (3HP; $n=31$ ) without a significant difference between the two groups. Other studies did not provide data with a control group [4, 16-18].

\section{Pregnancy outcomes}

Four studies provided data on the comparison between IPT and no treatment or a placebo among pregnant women living with HIV (supplementary table S6). All of them reported composite pregnancy outcomes including at least low birth weight, preterm delivery, spontaneous abortion, stillbirth and major congenital anomaly in the composite. KALK et al. [15] additionally included termination of pregnancy and neonatal death, while TAYLOR et al. [17] included neonatal death. The frequency of these composite pregnancy outcomes among women given IPT ranged from $15.0 \%$ to $31.1 \%$. In three studies reporting the frequency of individual outcomes, prematurity and low birth weight were commonly observed in women given IPT (10.1-13.4\% for prematurity and $8.7-14.9 \%$ for low birth weight) $[5,13,15]$. Results were similar in women not exposed to IPT (supplementary table S6).

Results from the one RCT and three NRS were inconsistent (figure 2). The RCT showed a significantly higher risk of composite adverse pregnancy outcomes in those who initiated IPT during pregnancy

\begin{tabular}{|c|c|c|c|}
\hline Study & IPT & Control & Risk ratio $(95 \% \mathrm{CI})$ \\
\hline GuPTA et al. [5] & 1 out of $477(0.2 \%)$ & $\begin{array}{c}\text { Placebo } \\
3 \text { out of } 479(0.6 \%)\end{array}$ & $0.33(0.03-3.21)$ \\
\hline KALK et al. [15] & 18 out of $10715(0.2 \%)$ & $\begin{array}{c}\text { No treatment } \\
103 \text { out of } 41227(0.3 \%)\end{array}$ & $0.67(0.41-1.11)$ \\
\hline SALAZAR-Austin et al. [13] & 0 out of $71(0 \%)$ & $\begin{array}{c}\text { No isoniazid exposure } \\
2 \text { out of } 84(2 \%)\end{array}$ & $0.24(0.01-4.84)$ \\
\hline TIAM et al. [16] & 2 out of $124(1.6 \%)$ & NA & NA \\
\hline TAYLOR et al. [17] & 0 out of $103(0 \%)$ & $\begin{array}{c}\text { No isoniazid exposure } \\
0 \text { out of } 93(0 \%)\end{array}$ & NA \\
\hline
\end{tabular}

IPT: isoniazid preventive therapy; $\mathrm{Cl}$ : confidence interval; NA: not available. 
$\mathrm{OR}(95 \% \mathrm{Cl})$

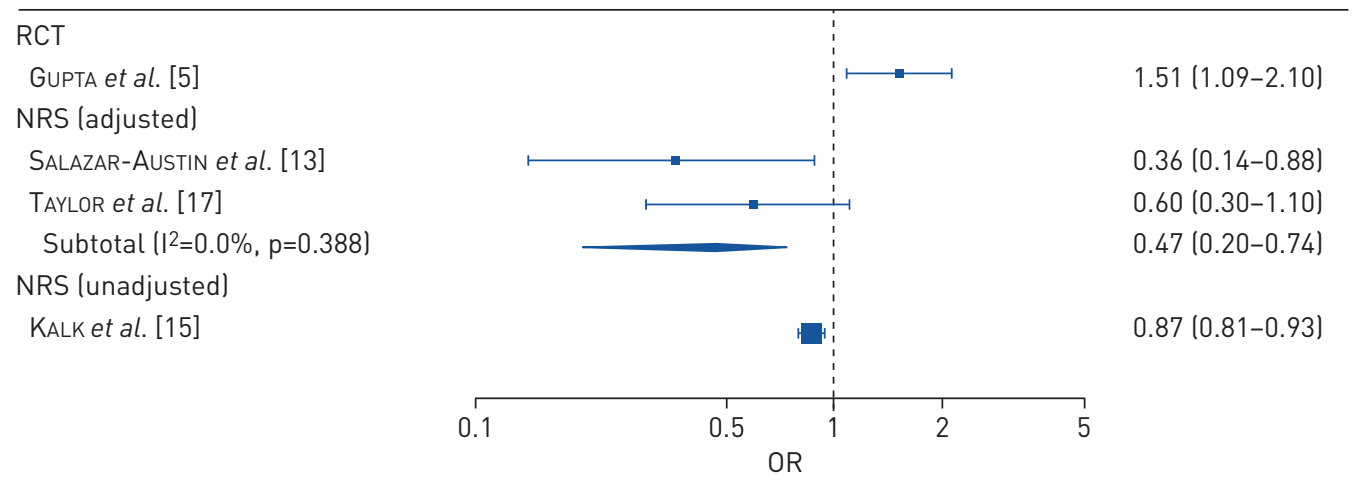

FIGURE 2 Composite pregnancy outcomes in pregnant women with HIV. A meta-analysis conducted using adjusted odds ratios (ORs). RCT: randomised controlled trial; NRS: non-randomised study; Cl: confidence interval.

(Mantel-Haenszel odds ratio (OR) stratified by gestational age; OR 1.51, 95\% CI 1.09-2.10), while a meta-analysis of composite outcomes using adjusted estimates from two NRS suggested a significantly lower risk of adverse pregnancy outcomes (OR 0.47, 95\% CI 0.20-0.74) (figure 2). Due to substantial heterogeneity $\left(\mathrm{I}^{2}=80 \%, \mathrm{p}=0.002\right)$, we did not pool data from the RCT and NRS.

A similar trend was observed when individual outcomes were analysed (figure 3 and supplementary tables S7-S10). In the RCT [5], women on IPT were more likely to experience still birth, spontaneous abortion, neonatal death, preterm birth, low birth weight, or congenital anomaly, although none of these were statistically significant. SALAZAR-AUSTIN et al. [13] reported a lower risk of low birth weight and preterm delivery in those given IPT, while, in the study by KALK et al. [15], IPT was significantly associated with lower risk of individual adverse pregnancy outcomes.

In HIV-negative pregnant women, only one study reported data on pregnancy outcomes (still birth, spontaneous abortion, neonatal death and congenital anomaly) [14]. This study did not find a statistical difference between pregnant women exposed to IPT, 3HP, or no treatment; however, the number of women in each group was very small $(n=56, n=31$ and $n=39$, respectively).

Quality of evidence assessment

Supplementary tables S1 and S2 in appendix 2 present the results of a risk of bias assessment. The risk of bias in the RCT by GUPTA et al. [5] was considered to be of some concern due to missing outcome data

IPT

No IPT

Risk ratio $(95 \% \mathrm{CI})$

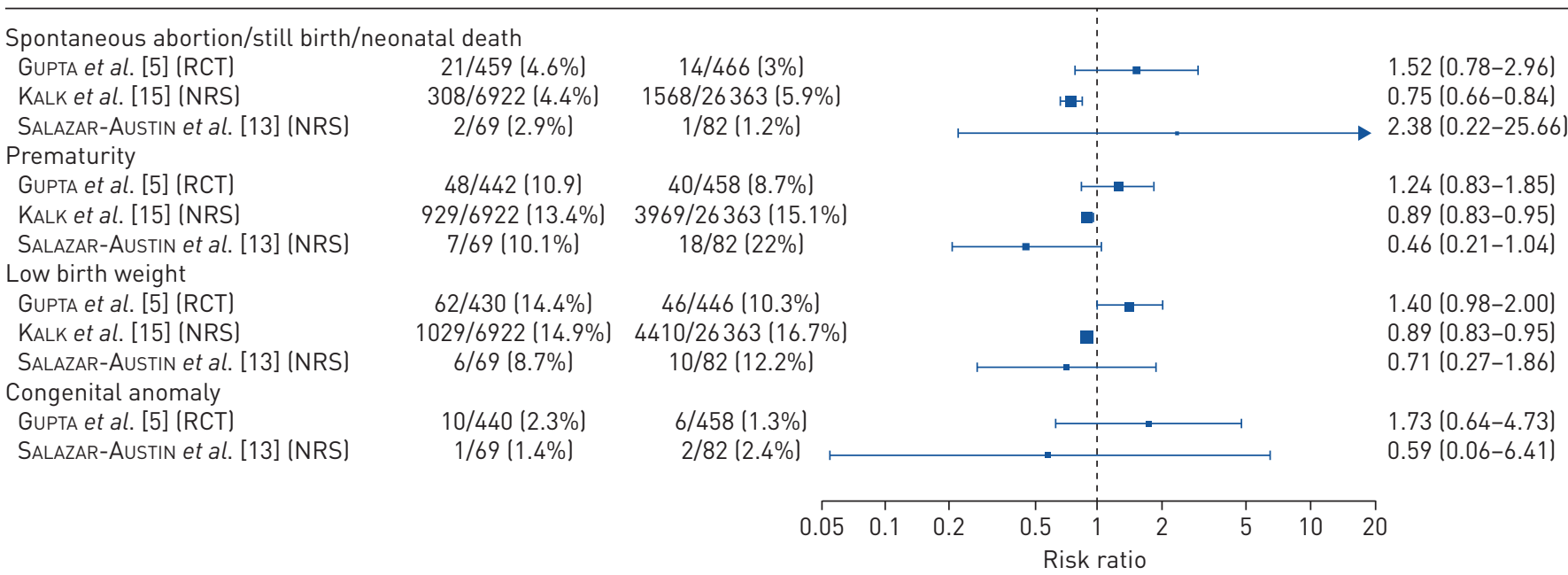

FIGURE 3 Individual pregnancy outcomes in pregnant women with HIV. IPT: isoniazid preventive therapy; Cl: confidence interval; RCT: randomised controlled trial; NRS: non-randomised study. 
(15.9\% in the immediate IPT arm and $17.3 \%$ in the deferred IPT arm). Of the four NRS with a control group that reported pregnancy outcomes, all were considered at serious risk of bias.

Quality of evidence was rated on a comparison between IPT and no preventive treatment or placebo among pregnant women with HIV (table 4). Certainty of evidence ranged from low to moderate for the one RCT and from very low to low for two NRS.

\section{Discussion}

This is the first systematic review that has evaluated the safety of IPT among pregnant women. Our review found inconsistent associations between IPT and adverse pregnancy outcomes among pregnant women with HIV in different studies. IPT was associated with more adverse pregnancy outcomes in one RCT while it was protective in three NRS. Frequency of hepatotoxicity was higher in the RCT than NRS.

There are several possible reasons for the discrepancy. First, it may be explained by differences in participant characteristics and settings. In the RCT, almost all of the participants $(99.8 \%$ in the immediate IPT arm and $100 \%$ in the delayed IPT arm) were already on combination antiretroviral therapy (cART) at baseline. However, this was not the case in the three NRS. In the study by SALAZAR-AusTin et al., $72 \%$ of women were on antiretroviral therapy (ART) at delivery, while in the study by TAYLOR et al., only $37 \%$

TABLE 4 Grading of recommendations assessment, development, and evaluation (GRADE) methodology in assessment of evidence regarding isoniazid preventive therapy (IPT) compared to no IPT or placebo for pregnant women living with HIV

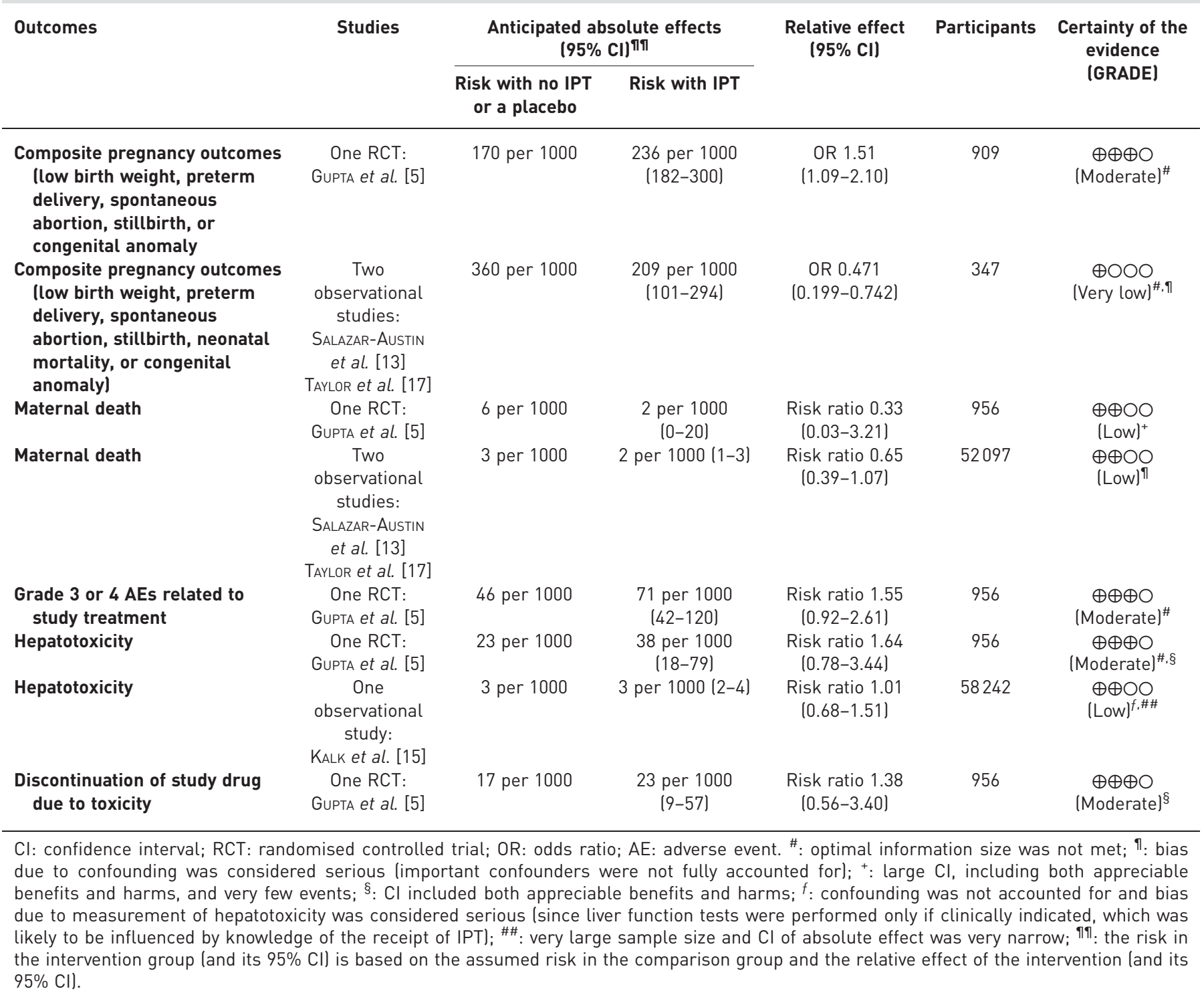


received cART during pregnancy. In the study by KALK et al., although all women were on cART, $41.8 \%$ of them started it during pregnancy. In fact, median CD4 counts were higher in the RCT study (491 cells $\mathrm{mm}^{-3}$ in the immediate IPT arm and 496 cells $\cdot \mathrm{mm}^{-3}$ in the deferred IPT arm) compared to the other studies with median CD4 counts ranging from $364-424$ cells $\cdot \mathrm{mm}^{-3}$. Furthermore, the three NRS were conducted in South Africa and Botswana, where TB incidence is estimated to be amongst the highest in the world [20]. In contrast, only one third of the RCT study subjects were enrolled in South Africa and Botswana and the remaining participants were from countries with a lower TB incidence. Women in the NRS may thus have been at a higher risk of TB than those in the RCT. In fact, KALK et al. reported a 1.5-fold higher risk of TB in those not given IPT. It is therefore possible that IPT reduced adverse pregnancy outcomes by averting more active TB during gestation. However, SALAZAR-AusTiN et al. reported no TB cases in the control group during pregnancy and the reason for reduction of adverse pregnancy outcomes in the IPT group thus remains unclear [13]. Secondly, NRS were at higher risk of bias. For example, they did not control for all important confounders such as history of liver disease, alcohol use and pregnancy history. Thirdly, it is possible that the RCT found more adverse pregnancy outcomes by chance.

The higher frequency of hepatotoxicity observed in the RCT could also be explained by a difference in the uptake of ART. ART causes hepatotoxicity and drug interactions while IPT may increase the risk further $[21,22]$. In addition, due to its study design, the RCT measured events that developed while participants were on placebo. Therefore, as the authors discuss, not all hepatotoxic events are attributable to IPT [5]. It is also likely that rigorous monitoring and systematic laboratory testing during follow-up may have detected more events than would be observed under routine programmatic conditions. The NRS performed liver function tests only when clinically indicated, in accordance with the standard practice recommended by the WHO $[13,15,17]$. Asymptomatic liver enzyme elevation may be transient or resolve after completion of treatment without causing clinically significant effects. It is unknown whether routine liver function testing actually prevents clinically significant hepatotoxicity through earlier cessation of a medicine in the field.

Given the findings from the one RCT, deferral of preventive TB treatment may be justifiable in those with low risk for TB after careful consideration of benefits and harms and informed choice of the woman. However, this needs caution as multiple studies have reported loss to HIV-care after delivery [23-25]. Therefore, deferral of IPT may lead to a missed opportunity to protect women and their babies from TB and death. Although data on IPT are limited in pregnant women, this should not be an impediment to giving preventive $\mathrm{TB}$ treatment to pregnant women at high risk for progression to active TB. To strengthen confidence in initiating preventive TB treatment during pregnancy, safety studies are needed in both HIV-positive and HIV-negative pregnant women. These studies should include different regimens and would preferably be designed as RCTs of appropriate power to measure key pregnancy and maternal outcomes individually. Pooled meta-analysis from person-level data that includes longer-term postpartum surveillance for AEs in infants would also be helpful.

The strengths of this review include the use of a comprehensive search strategy, explicit inclusion criteria, a systematic approach to data collection and an independent assessment for study inclusion and data extraction. This enabled the first comprehensive assessment of the body of evidence on the safety of IPT among pregnant women. Our review revealed that the increased risk of adverse pregnancy outcomes due to IPT in a single RCT was not supported by multiple NRS, although their risk of bias was serious. This finding and the limited number of studies available signal an urgent need for more research on this important clinical and public health issue.

This review has several limitations. First, the majority of studies that met the inclusion criteria were among pregnant women living with HIV and who were aware of their status. Only three studies provided data on IPT safety among HIV-negative pregnant women and pregnant women living with HIV who were unaware of their status. Of these, two did not include a control group that was not given IPT. Furthermore, the associations observed among HIV-positive pregnant women are likely to be influenced by the concurrent use of ART and the increased risk of developing TB in these women and thus the findings are not fully generalisable to HIV-negative pregnant women. Secondly, limited data were available on the safety of IPT compared to rifamycin-containing preventive TB regimens among pregnant women. Thirdly, our primary analysis focused on composite adverse pregnancy outcomes because adjusted ORs were not available for individual outcomes. The composite outcome was driven by preterm delivery and low birth weight. The frequency of other outcomes (e.g. congenital anomaly and still birth) is usually much lower than those outcomes [26-29]. This was also the case in our review and hence less evidence is available on the impact of IPT on the other outcomes.

In conclusion, a single RCT showed an increased risk of adverse pregnancy outcomes due to IPT, while three NRS suggested it has a protective effect. The benefits of IPT may outweigh its potential AEs in 
women at high risk of TB. Therefore, our findings do not support systematic deferral of IPT until postpartum regardless of the risk of TB.

Acknowledgements: We gratefully acknowledge valuable inputs to our protocol and report from Nathan Ford (Dept of HIV/AIDS and Global Hepatitis Programme, World Health Organization, Switzerland), Nandi Siegfried (individual consultant, South Africa) and Lynne M. Mofenson (Elizabeth Glaser Pediatric AIDS Foundation, USA). We also thank Amita Gupta and Nicole Salazar-Austin (Johns Hopkins University, USA) for providing additional unpublished data. D. Falzon, C. Figueroa and A. Kanchar are employees of the World Health Organization (WHO). They alone are responsible for the views expressed in this publication, which do not necessarily represent the decisions or policies of the WHO. Furthermore, the designations used in this publication and the manner of presentation of the material do not imply any opinion whatsoever on the part of the WHO concerning the legal status of any country, territory, city or area, or of its authorities, or the delimitation of its frontiers or boundaries.

Conflict of interest: None declared.

\section{References}

1 Gupta A, Nayak U, Ram M, et al. Postpartum tuberculosis incidence and mortality among HIV-infected women and their infants in Pune, India, 2002-2005. Clin Infect Dis 2007; 45: 241-249.

2 World Health Organization. Guidelines for intensified tuberculosis case-finding and isoniazid preventive therapy for people living with HIV in resource-constrained settings. Geneva, Switzerland, WHO, 2011.

3 World Health Organization. Latent TB infection: updated and consolidated guidelines for programmatic management. Geneva, Switzerland, WHO, 2018.

4 Franks AL, Binkin NJ, Snider DE Jr, et al. Isoniazid hepatitis among pregnant and postpartum Hispanic patients. Public Health Rep 1989; 104: 151-155.

5 Gupta A, Montepiedra G, Aaron L, et al. Isoniazid preventive therapy in HIV-infected pregnant and postpartum women. N Engl J Med 2019; 381: 1333-1346.

6 Liberati A, Altman DG, Tetzlaff J, et al. The PRISMA statement for reporting systematic reviews and meta-analyses of studies that evaluate healthcare interventions: explanation and elaboration. BMJ 2009; 339: b2700.

7 Stroup DF, Berlin JA, Morton SC, et al. Meta-analysis of observational studies in epidemiology: a proposal for reporting. JAMA 2000; 283: 2008-2012.

8 Higgins JPT, Sterne JAC, Savović J, et al. A revised tool for assessing risk of bias in randomized trials. In: Chandler J, McKenzie J, Boutron I, et al., eds. Cochrane Methods. Cochrane Database of Systematic Reviews 2016; 10 (Suppl 1).

9 Sterne JA, Hernán MA, Reeves BC, et al. ROBINS-I: a tool for assessing risk of bias in non-randomised studies of interventions. BMJ 2016; 355: i4919.

10 Guyatt G, Oxman AD, Akl EA, et al. GRADE guidelines: 1. Introduction-GRADE evidence profiles and summary of findings tables. J Clin Epidemiol 2011; 64: 383-394.

11 Schunemann HJ, Cuello C, Akl EA, et al. GRADE guidelines: 18. How ROBINS-I and other tools to assess risk of bias in nonrandomized studies should be used to rate the certainty of a body of evidence. J Clin Epidemiol 2019; 111: 105-114.

12 DerSimonian R, Laird N. Meta-analysis in clinical trials. Control Clin Trials 1986; 7: 177-188.

13 Salazar-Austin N, Cohn S, Lala S, et al. Isoniazid preventive therapy and pregnancy outcomes in HIV-infected women in the Tshepiso cohort. Clin Infect Dis 2019; in press [https://doi.org/10.1093/cid/ciz1024].

14 Moro RN, Scott NA, Vernon A, et al. Exposure to latent tuberculosis treatment during pregnancy. The PREVENT TB and the iAdhere Trials. Ann Am Thorac Soc 2018; 15: 570-580.

15 Kalk EK, Heekes A, Mehta U, et al. Programmatic review of safety and effectiveness of isoniazid preventive therapy in HIV-infected pregnant women on ART in routine care. Reprod Toxicol 2018; 80: 155.

16 Tiam A, Machekano R, Gounder CR, et al. Preventing tuberculosis among HIV-infected pregnant women in Lesotho: the case for rolling out active case finding and isoniazid preventive therapy. J Acquir Immune Defic Syndr 2014; 67: e5-e11.

17 Taylor AW, Mosimaneotsile B, Mathebula U, et al. Pregnancy outcomes in HIV-infected women receiving long-term isoniazid prophylaxis for tuberculosis and antiretroviral therapy. Infect Dis Obstet Gynecol 2013; 2013: 195637.

18 Chang AH, Polesky A, Bhatia G. House calls by community health workers and public health nurses to improve adherence to isoniazid monotherapy for latent tuberculosis infection: a retrospective study. BMC Public Health 2013; 13: 894 .

19 Msandiwa R, Barnes G, Ram M, et al. Pregnancy in HIV-infected women in a tuberculosis preventive therapy trial (abstract TUPEB141). In: IAS Conference of HIV Science; 2009; Cape Town, South Africa, 2009.

20 World Health Organization. Global tuberculosis report 2018. Geneva, Switzerland, WHO, 2018.

21 Shubber Z, Calmy A, Andrieux-Meyer I, et al. Adverse events associated with nevirapine and efavirenz-based first-line antiretroviral therapy: a systematic review and meta-analysis. AIDS 2013; 27: 1403-1412.

22 Dooley KE, Denti P, Martinson N, et al. Pharmacokinetics of efavirenz and treatment of HIV-1 among pregnant women with and without tuberculosis coinfection. J Infect Dis 2015; 211: 197-205.

23 Phillips T, Thebus E, Bekker LG, et al. Disengagement of HIV-positive pregnant and postpartum women from antiretroviral therapy services: a cohort study. J Int AIDS Soc 2014; 17: 19242.

24 Adams JW, Brady KA, Michael YL, et al. Postpartum engagement in HIV care: an important predictor of long-term retention in care and viral suppression. Clin Infect Dis 2015; 61: 1880-1887.

25 Swain CA, Smith LC, Nash D, et al. Postpartum loss to HIV care and HIV viral suppression among previously diagnosed HIV-infected women with a live birth in New York state. PLoS One 2016; 11: e0160775.

26 Dolk H, Loane M, Garne E. The prevalence of congenital anomalies in Europe. Adv Exp Med Biol 2010; 686: 349-364. 
27 GBD 2015 Child Mortality Collaborators. Global, regional, national, and selected subnational levels of stillbirths, neonatal, infant, and under-5 mortality, 1980-2015: a systematic analysis for the Global Burden of Disease Study 2015. Lancet 2016; 388: 1725-1774.

28 Blencowe $\mathrm{H}$, Cousens S, Oestergaard MZ, et al. National, regional, and worldwide estimates of preterm birth rates in the year 2010 with time trends since 1990 for selected countries: a systematic analysis and implications. Lancet 2012; 379: 2162-2172.

29 Blencowe H, Krasevec J, de Onis M, et al. National, regional, and worldwide estimates of low birthweight in 2015, with trends from 2000: a systematic analysis. Lancet Glob Health 2019; 7: e849-e860. 